\title{
LA NOCIÓN DE ‘EMOCIÓN’: DE LA FILOSOFÍA MORAL AL ESTUDIO CIENTÍFICO ${ }^{1}$
}

\author{
The Notion of "Emotion": From a Moral Philosophy to a Scientific Study
}

A noção de 'emoção': da filosofia moral ao estudo científico

RECIBIDO: 13 DE FEBRERO DE 2015

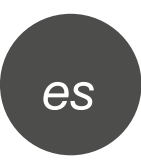

\section{RESUMEN}

El desarrollo científico en campos como la psicofisiología o la biología y la inquietud que pensadores como Charles Darwin, William James o Wilhelm Wundt sintieron por el concepto 'emoción' hicieron que el uso lingüístico de esta palabra se modificara de manera definitiva. Durante el siglo XIX dicho concepto pasó de ocupar un lugar exclusivo en el discurso teológico y moral a ser un concepto central en la consolidación de las nuevas disciplinas científicas que competen a lo humano. En Colombia, el arribo de la psicología experimental traería consigo esa nueva forma de entender la emoción. El presente artículo es una revisión bibliográfica de los autores mencionados que, durante el siglo XIX, fueron decisivos para propiciar dicho cambio

PALABRAS CLAVE: emoción, concepto, William James, psicología experimental, psicofisiología.

\section{EVALUADO:13 DE ABRIL DE 2015}

\author{
Carlos Andrés Salazar Martínez (Colombia) \\ Doctorando en Ciencias Humanas, Magíster \\ en Hermenéutica Literaria \\ Universidad Eafit \\ casalazar@gmail.com
}

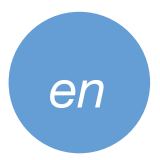

\section{ABSTRACT}

The scientific development in fields like psychophysiology or biology and the curiosity of thinkers such as Charles Darwin, William James, or Wilhelm Wundt regarding the concept of "emotion" caused the change of the linguistic use of this word definitively. During the XIX century, this concept went from occupying an exclusive place in the theological and moral discourse to being a central concept in the consolidation of new scientific disciplines that belong to the human being. In Colombia, the experimental psychology introduced this new way to understand emotion. This article is a bibliographic revision of those authors that were essential for this change during the XIX century.

KEYWORDS: emotion, concept, William James, experimental psychology, psychophysiology.

\section{RESUMO}

O desenvolvimento científico em campos como a psicofisiologia ou a biologia e a inquietação que pensadores como Charles Darwin, William James ou Wilhelm Wundt sentiram pelo conceito 'emoção' fizeram que o uso linguístico de esta palavra se modificara de maneira definitiva. Durante o século XIX dito conceito passou de ocupar um lugar exclusivo no discurso teológico e moral a ser um conceito central na consolidação das novas disciplinas científicas que competem ao humano. Na Colômbia, a chegada da psicologia experimental traria consigo essa nova forma de entender a emoção. $\mathrm{O}$ apresente artigo é uma revisão bibliográfica dos autores mencionados que, durante o século XIX, foram decisivos para propiciar dito câmbio.

PALAVRAS CHAVE: emoção, conceito, William James, psicologia experimental, psicofisiologia.

PARA CITAR ESTE ARTÍCULO/TO CITE THIS ARTICLE/PARA CITAR ESTE ARTIGO: Salazar Martínez, C. A. (2015). La noción de 'emoción': de la filosofía moral al estudio científico. Panorama, 9(16), 56-64. 


\section{INTRODUCCIÓN}

Los avances científicos en neurobiología (Changeux y Ricoeur, 2001) y los actuales estudios en disciplinas como la economía conductual (Kahneman, 2012) o la toma de decisiones (Brooks, 2012) han renovado y vuelto a poner en contexto las preguntas en torno a las emociones. Se suma a esto un creciente interés por el desarrollo de modelos matemáticos y dispositivos que permitan su detección. Ya sea en reconocimiento de imágenes considerando la teoría de la expresión facial de las emociones del psicólogo estadounidense Paul Ekman (1979, 1984, 2009), ya sea en reconocimiento de patrones en señales de audio de acuerdo con la teoría de la expresión vocal de las emociones de Scherer (1981, 1991, 2000, 2003, 2005, 2009). Desde aquí es importante considerar la manera en que el significado de un concepto como 'emoción' se ha amoldado para ser entendido desde la filosofía moral o teológica hasta la psicología o la biología. Cada uno de estos puntos de vista ha ofrecido, a su manera, respuestas a la pregunta: ¿qué es una emoción?

Veinticinco años después de la publicación de $E l$ origen de las especies, Charles Darwin haría contribuciones fundamentales para comprender cómo el ser humano manifiesta sus emociones. En su libro La expresión de las emociones en el hombre y los animales (1872/1984), trata las emociones como entidades discretas o modulares, tales como la ira, la sorpresa o el miedo. El aporte de este libro sería fundamental en la transformación que sufriría la noción de 'emoción'. Incluso, teniendo como fuente primaria el aporte de Darwin, sería que Ekman y Oster (1979), para la década de 1970, demostraría que hay emociones cuya expresión e identificación es de carácter universal. Es decir, hay emociones que, más allá de condicionamientos culturales, son representadas e interpretadas según ciertas consideraciones biológicas.

Eric Kandel (2001), por ejemplo, autor del libro Principios de neurociencia y Premio Nobel de Medicina, señala que el aporte de William James y Carl Lange fue decisivo para abrir un debate mucho más amplio respecto de esta cuestión. A finales del siglo XIX, suscitaría un gran interés la famosa frase de James según la cual "Estamos tristes porque lloramos, nos enfurecemos porque golpeamos, tenemos miedo porque temblamos, y no lloramos, golpeamos o temblamos porque estemos tristes, enfurecidos o temerosos según sea el caso" (2001, p. 963-964). Esta teoría sería el detonante para que la psicofisiología avanzara en una línea que luego de un siglo, y con otro nombre, le ha permitido determinar que una emoción es el resultado de una interacción dinámica entre factores extrínsecos e intrínsecos a la propia conciencia. Antonio Damasio (2010, p. 43), neurobiólogo contemporáneo, define de manera específica las emociones como el mecanismo que apunta "directamente a la regulación vital a fin de evitar los peligros o ayudar al organismo a sacar partido de una oportunidad, o indirectamente a facilitar las relaciones sociales".

A principios del siglo XX, Wilhelm Wundt (1905) estudiaría características adicionales respecto de la expresión de una emoción, y es que estas, según el psicólogo alemán, pueden ser reconocidas por el grado de valencia y por las variaciones en la excitación que cada una de ellas produce en los individuos. James Russell (1980) propondría, luego, una teoría en la que los estados emocionales pueden representarse como puntos de un círculo en un espacio bidimensional. Este plano considera bipolaridades que se señalan por medio del siguiente orden: placer $\left(0^{\circ}\right)$, excitación $\left(90^{\circ}\right)$, fastidio $\left(180^{\circ}\right)$, reposo $\left(270^{\circ}\right)$. Así es como, aparte de ubicar en dicho plano las siete emociones básicas identificadas por Ekman (felicidad, sorpresa, desdén, tristeza, ira, asco y miedo), Russell dio cabida a veintiún emociones más, entre las que se cuentan: la frustración, la calma, el aburrimiento, la angustia, y demás. La importancia de esta medida radica en que permite establecer relaciones entre los componentes de bienestar y excitación con coordenadas específicas en un plano bidimensional de emociones. En este plano es posible establecer relaciones de causalidad para mapear un espacio de bienestar y excitación. Al hacer esto, Russell permitió concebir las emociones como magnitudes y, a su vez, abriría el camino para que diferentes ramas de las matemáticas pudieran dar explicaciones sobre la generación de emociones y las interrelaciones entre mecanismos neuronales y psicológicos. Como puede observase, y pese a ser común leer simplemente que la palabra emoción procede del latín e-movere, 'moverse hacia', para cada generación ha marcado un profundo desafío conocer y describir las causas de ese movimiento; entender el cómo y el porqué se genera. Así es como cada vez que se han presentado nuevas soluciones a estos interrogantes, y de manera puntual debido a la participación activa de la ciencia en el siglo XIX, se modifica el sentido y el uso lingüístico de este concepto. Reconocer que la palabra emoción tiene
La noción de ‘emoción': de la 
un registro que cambia con la historia implica investigar de qué manera se han dado dichas variaciones.

\section{PARA UNA HISTORIA DEL CONCEPTO 'EMOCIÓN'}

Carlos Andrés

Salazar

Martínez I

En la introducción al libro historia/Historia (2010), Antonio Gómez Ramos pone en evidencia el método por medio del cual Reinhart Koselleck lleva con acierto la reconstrucción histórica de conceptos como 'crisis' e 'historia'. Koselleck, estudiante de Gadamer, utilizaría la hermenéutica alemana de la posguerra para llamar la atención de los historiadores respecto del hecho de que "las palabras tienen una vida propia que se escapa a cualquier encasillamiento de diccionario, y que los conceptos dibujados con esas palabras son igualmente sujetos de una historia que queda si se ofrece una imagen fija" (2010, p. 12). Para Koselleck, sin embargo, hacer una historia de los conceptos requiere llevar a cabo una historia social, ya que exige

$$
\begin{aligned}
& \text { analizar cómo la entrada en acción de } \\
& \text { los conceptos, su uso, provoca unas } \\
& \text { transformaciones estructurales que afectan } \\
& \text { tanto a la sociedad como al concepto } \\
& \text { mismo. Pues tales transformaciones, que } \\
& \text { son la historia misma, constituyen a la par } \\
& \text { la realidad del concepto que se estudia. } \\
& \text { Es decir, es preciso un estudio sincrónico } \\
& \text { de los conceptos que se combine con un } \\
& \text { principio diacrónico que siga la sucesión de } \\
& \text { los significados de las palabras y permita así } \\
& \text { una traducción a nuestra compresión actual } \\
& \text { (p. 16). }
\end{aligned}
$$

Por eso, la historia conceptual oscila entre el concepto y el estado de cosas que este describe, en el puente entre las situaciones sociales reales y el uso lingüístico que se refiere a ellas. "Su método alterna la semasiología, atendiendo a los múltiples significados que se dan para un mismo término, con la onomasiología, considerando las múltiples denominaciones que existen para estados de cosas en principio idénticos" (p. 18). Muy en la vía de la propuesta metodológica de Koselleck, y que Gómez Ramos hace emerger en su reflexión, los pioneros de la historia de las emociones plantean que su experiencia, hasta el momento, indica:

Para alcanzar su total potencial, la historia de las emociones necesita tomar entre sus cuentas la visión que surja desde la semántica cultural. Hay un creciente interés en reconocer el lenguaje como un asunto clave

para entender las emociones humanas y en el hecho de que, adicionalmente, el manejo de los términos relacionados con la emoción en inglés, como herramientas analíticas, es un gran obstáculo para el progreso de la investigación y su entendimiento (Wierzbicka, 2010, p. 272).

Este interés permite ampliar la complejidad en el estudio de un concepto como el que ocupa este trabajo debido a que no puede entenderse si se le aísla de términos que le son cercanos; de la misma manera que Koselleck reconoce, por ejemplo, que para hacer un análisis del concepto de 'historia' debía incluir conceptos complementarios, como 'revolución', 'azar', 'destino', 'progreso' y 'desarrollo' (Koselleck, 1993, p. 17). Tanto para Koselleck como para los historiadores de las emociones, las fuentes de las cuales se valen para reconstruir el contexto sociohistórico en el que se define cada uno de los conceptos son múltiples, una de ellas es la literatura. Susan Matt (2011) y Peter Stearns (2014), dos importantes figuras en la naciente disciplina de la historia de las emociones, recopilan algunos ejemplos respecto de los estudios que se han realizado considerando estas fuentes ${ }^{1}$, no sin precisar que todas ellas tienen la misma limitación, y es que "revelan mucho de los artistas, escritores, los psicólogos y la manera en que los expertos entienden el mundo, pero ellas no pueden revelar si tales puntos de vista y sentimientos fueron ampliamente difundidos” (Matt y Stearns, 2013, p. 49).

Para comprender, por tanto, qué sucede con la noción de 'emoción' y esa relación que estableció, en algún punto de sus variaciones semánticas, con la ciencia es pertinente reconstruir parte de su historia. En este punto, se debe resaltar el hecho de que fue con la literatura que la palabra llegó al Reino Unido. Es importante considerar esto, ya que, serían los ingleses y, posteriormente, los estadounidenses, quienes darían al concepto el giro hacia dicha perspectiva. Así lo describe Thomas Dixon:

La palabra emoción arribó primero al Reino
Unido desde Francia a principios del siglo
XVII.John Florio, el traductor de los ensayos
de Michel de Montaigne, pidió disculpas a
sus lectores por la introducción de varios
"términos toscos" del francés en su versión
inglesa, incluyendo entre ellos la palabra

1 Entre estos ejemplos destaca el que William Reddy (2001) haya utilizado los ensayos de Voltaire y las novelas de Balzac y Sand para mostrar cómo han cambiado los estados de ánimo emocionales en Francia. 
emoción" ${ }^{2}$, . Tanto en su forma para el inglés como para el francés, emoción fue una palabra utilizada para denotar los disturbios físicos y los movimientos corporales. Podría significar entonces una conmoción entre un grupo de personas (como en la frase "la emoción del público"), o una agitación física de cualquier cosa, desde el tiempo o un árbol, en el cuerpo humano (2012, p. 340).

En el siglo XIX, el concepto tendría una nueva transformación. Thomas Brown (1820/2010), profesor de Filosofía Moral en Edimburgo y a quien Dixon llama "el inventor de las emociones", recoge los "apetitos", las "pasiones" y las "afecciones" bajo una misma categoría: las "emociones" (Dixon, 2012, p. 340). Es con Brown con quien comienza a revelarse la necesidad de crear un campo semántico particular para el concepto 'emoción'. Muchos de esos otros conceptos enumerados proceden de la tradición moral y escolástica, pero sabría ver Brown en ellos una afinidad con la palabra emoción. Concepto que, hasta ese momento, se utilizaba para designar los cambios en el estado del cuerpo.

La segunda figura clave fue otro filósofo y fisiólogo de Edimburgo, Charles Bell. Bell fue una figura importante en la historia de la neurología y uno de los teóricos más influyentes del siglo XIX respecto de las expresiones antes de 1872, cuando Darwin publica su trabajo sobre este tópico. Las teorías de las emociones y las expresiones de Bell fueron elaboradas y expuestas en las sucesivas ediciones de sus ensayos de anatomía y filosofía de la expresión publicados entre 1806 y 1844, con lo cuales proporsionó las fundaciones sobre las que construirían sus propias teorías Darwin y James (Dixon, 2012, p. 341).

El uso moderno de los conceptos 'emoción' y 'emocional' es heredero de la simpatía que existía por el enfoque científico de la vida mental en los seres humanos durante el siglo XIX. Ejemplo de ello es que en los diccionarios de la Real Academia Española la palabra

2 Esta afirmación hace pensar, inevitablemente, que es necesario explorar cómo llegó, por primera vez, al español el concepto 'emoción'. Verificar si, quizá, su llegada obedece, también, a la influencia de autores franceses, como Montaigne.

3 En español, por ejemplo: "Hay tantos medios de evitar individualmente las ocasiones de exponerse, que podemos engañar mil veces al mundo antes de poner el pie en un lugar donde el peligro nos amenace; y aun entonces, encontrándonos entre la espada y la pared, sabremos ocultar las emociones de nuestro rostro, expresándonos con palabra serena, aunque nuestra alma vacile interiormente" (Montaigne, 2003, cap. XVI).

4 El concepto tradicional de 'pasión', en la filosofía moderna, es ilustrado por Descartes en Las pasiones del alma (Deigh, 2014, p. 7). emoción comienza a ser registrada a partir de 1884 . Y se observa cómo a partir de ahí su significado ha sido sometido a cambios drásticos.

- 1884. Emoción: agitación repentina del ánimo.

- 1925. Emoción: agitación del ánimo que promueve en él afectos o pasiones.

- 1992. Emoción: estado de ánimo producido por impresiones de los sentidos, ideas o recuerdos que con frecuencia se traduce en gestos actitudes, $\mathrm{u}$ otras formas de expresión.

- 2001. Emoción: alteración del ánimo intensa y pasajera, agradable o penosa, que va acompañada de cierta conmoción somática. Interés expectante con que se participa en algo que está ocurriendo.

Como se puede observar para cada uno de los significados presentados con cada nueva edición, de 1884 en adelante se pone en juego la cercanía del concepto con alguna perspectiva específica: en 1925, por ejemplo, se continúa hablando de afectos o pasiones para definirlo. Sin embargo, antes de que la palabra apareciera, incluso en los diccionarios para el español, ya desde el inglés estaba siendo sometida a una resemantización, que la haría cambiar de registro y provoca transformaciones a otros niveles. De hecho, afirma Dixon (2012, p. 342), el cambio lingüístico fue tan significativo que provocó cambios importantes en lo institucional e intelectual. Sería a finales del siglo XIX que esta perspectiva llegaría a universidades de Europa y América, las cuales comenzaron a hacer, justo en ese momento, investigación científica de la mente humana con un estudio de fondo fisiológico.

Es a partir de este punto que William James (1980, 1984, 1994) y Carl Lange (1887) hacen el relevo en la carrera por definir el concepto de emoción desde una consideración fisiológica. La influencia de la esfera psicológica se extendió hasta 1930, debido a que a partir de ahí se dio el ascenso del conductismo, perspectiva que se abstiene de considerar los estados subjetivos internos. Con el pragmatismo, sostiene Eugene Taylor (2010, pp. 413-414), William James llamó la atención de filósofos de todas partes del mundo (John Dewey ${ }^{5}$, Josiah Royce, Friedrich Schiller, Henri Bergson y Giovanni Pappini), por lo que fue la primera corriente filosófica estadounidense de una resonancia mundial destacada. A partir de

5 Contrastando la teoría de Darwin y la de James-Lange, Dewey decide inclinarse por preferir la segunda propuesta debido, en parte, a que esta se enfoca en el movimiento o la respuesta funcional (Garrison, 2003, p. 406). 
ahí, las consideraciones desde la psicología funcionalista de James alcanzaron una resonancia mayor.

James fue uno de los progenitores de la escuela funcionalista desde el pensamiento psicológico, y sus Principios fueron de una gran influencia para prominentes funcionalistas, como John Dewey y James Rowland Angell. Estos pragmáticos, a diferencia de la aproximación psicológica estadouniense, guiados por los principios darwinianos, pusieron énfasis en la función adaptativa de la conciencia como la expresión de la relación mente-cuerpo. La teoría de las emociones de James fue profundamente influenciada por el libro La expresión de las emociones en hombres y animales, de Darwin. Esta perspectiva domina los Principios, los cuales ponen el ritmo para la psicología en América y tuvo una fuerte influencia en este campo (Friedman, 2010, p. 384).

La teoría de James-Lange (1922) completó la transformación del estudio de las emociones desde la filosofía moral al estudio científico ${ }^{6}$ (Deigh, 2014, p. 4). De hecho, el ataque que James hace en su libro Principios de psicología al esquema conceptual de los estados mentales característico del empirismo clásico británico y al esquema psicológico de Locke y Hume permite entender la importancia revolucionaria de la teoría funcionalista de las emociones (Deigh, 2014, p. 9).

\section{EL CONCEPTO 'EMOCIÓN'Y SU PERSPECTIVA CIENTÍFICA EN COLOMBIA}

Describir el punto en el que las teorías funcionalistas de la emoción compiten o pretenden reemplazar las perspectivas moralistas, escolásticas o empiristas es clave para estudiar cómo fueron adoptadas en un lugar como Colombia. Así lo describe Gilberto Oviedo, autor de la tesis de doctorado El proceso secular de la conciencia psicológica en Colombia (1886-1917):

\footnotetext{
Panorama I pp. 56-64। Volumen 9 । Número 16 | Enero-junio I 2015 I 6 Las preguntas que se abren respecto de considerar la teoría de James una teoría científica se responden en las cuestiones que propone Reisenzein y Stephan (2014), desde la perspectiva de Lakatos y su filosofía de la ciencia: “¿Ha producido la teoría de las emociones de James un programa de investigación progresivo, como afirman algunos, o se ha llevado a un callejón sin salida la investigación de las emociones, como han sugerido otros? Y ¿la teoría y el discurso científico que generó producen percepciones, ideas y argumentos que siguen siendo valiosos incluso si — como muchos sostienen— la teoría es falsa? (pp. 35-36).
}

produjeron ejemplos clave en la interacción entre el pensamiento psicológico y el proceso político en la construcción de Colombia como nación (2012, p. 293).

Luego de la Independencia, la lucha por la secularización de los asuntos humanos fue una de las empresas más importantes y serían los sectores políticos los que tendrían la última palabra en cuanto a qué tendencias psicológicas debían ser consideradas y puestas en práctica. De esta manera, cada una de las aspiraciones partidistas contribuiría para establecer un modelo de la psique en Colombia. Entre 1880 y 1894, durante la Presidencia de Núñez, se tomarían medidas para intentar abolir el régimen federalista y restaurar así el modelo centralista y teocrático. La Iglesia, por tanto, tendría un lugar privilegiado para discutir cuestiones políticas concernientes a la academia y la ciencia. Sin embargo, durante ese mismo periodo:

Ernesto Röthlisberger, en su prólogo al libro
Filosofía experimental, de César Guzmán,
expresó la importancia de una visión
materialista de los estados mentales a través
de la psicología científica. El intelectual
moderno debería concentrar su capacidad de
observación y de investigación en hechos y
fenómenos y alejarse de la metafísica para
dedicarse a la psicología, estudio reflexivo de la
naturaleza bumana y la expresión de la mente
y la psique. El sensacionalismo [empirismo]
tiene, para el liberalismo, el valor de una
posición teórica que facilita el acceso a una
nueva psicología, y en ella se pusieron las
esperanzas para romper con el peso de la
tradición escolástica (p. 295).

Debe destacarse entre las conclusiones de Gilberto Oviedo que considere de gran importancia para Colombia entender las suposiciones conceptuales e intereses que desencadenó la incorporación de la psicología experimental. Y, adicional a ello, el valor que implica para la historia reconocer la interacción de la política y la ciencia en la creación y el manejo de esa moderna nación Estado. En Colombia, más que William James, sería Wilhelm Wundt, fundador de la psicología experimental alemana, quien revolucionaría la concepción lingǘstica de conceptos no solo como conciencia, sino también como emoción.

Wundt concibió el concepto 'emoción' como el pilar para el desarrollo psíquico y como un componente esencial de la cognición (Wassmann, 2009, p. 224). De 
hecho, según Wassmann (2014, p. 168), William James reconoció que su interés en la cuestión de la naturaleza de la emoción procede del estudio de las primeras obras de Wilhelm Wundt. En la última década del siglo XIX, James y Wundt se enfrascarían en una discusión por el proceso exacto con el cual las señales periféricas o el sistema nervioso central generan estados emocionales. Así lo explica Palencik:

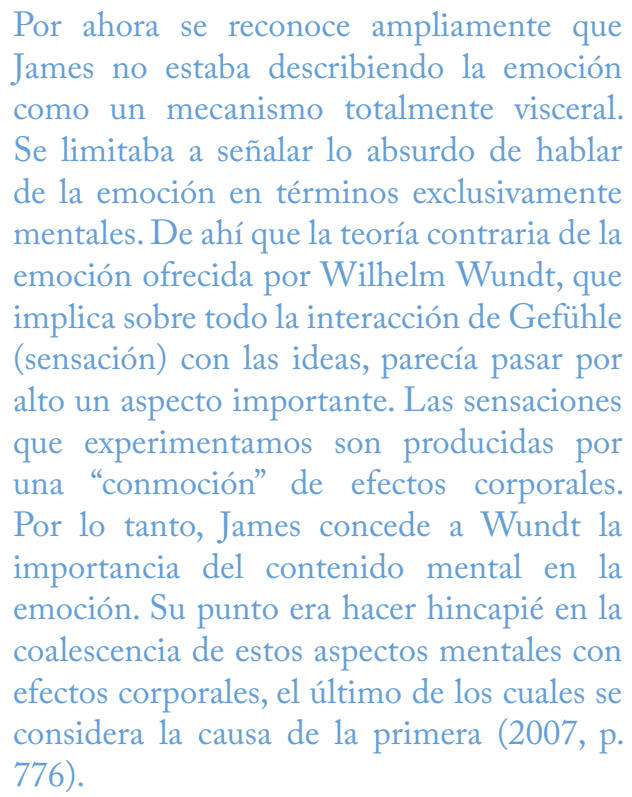

Considerar la cuestión de que la vertiente científica sobre la conciencia y las emociones ganaba terreno o, por lo menos, era reseñada en academias y universidades del país a finales del siglo XIX, implica atender a sus alcances, quizá desde ese enfrentamiento en el que se vio involucrada con la influencia política y religiosa presente en el país.

El debate que habría frente a cómo entender esas nuevas perspectivas no solo produciría confrontaciones políticas e ideológicas en los jóvenes Estados nación de América Latina. A su vez, pensadores como Bergson, debieron conciliar con su pensamiento la investigación empírica de la ciencia con la problematización metafísica de la filosofía (Costa, 2012, p. 134).

En 1889 Bergson (2007) publicaría en su tesis doctoral, L'essai sur les données immédiates de la conscience, reflexión en torno al papel que desempeña el cuerpo en la exteriorización de ciertas emociones, considerando las afirmaciones que hace al respecto Darwin (1872) en su libro La expresión de las emociones en hombres y animales. Bergson hace parte de otra visión que debe ser considerada en cuanto a la apropiación de estos conceptos a un contexto como el de Colombia de principios del siglo XX. Al estar su pensamiento centrado en el problema de cómo entender el diálogo entre la ciencia y la filosofía, sus afirmaciones parecían resolver los desacuerdos en los que se encontraban los pensadores y las escuelas de su época. Ante todo marcando la importancia que tiene el pensamiento filosófico respecto de los asuntos de la conciencia y el comportamiento humano. Debido a que, para Bergson, la ciencia solo puede proporcionar una visión parcial y limitada de la realidad, mucho menos puede entenderse que esté en capacidad de hacer una descripción precisa de las funciones intelectuales más elevadas de los seres humanos (Costa, 2012, p. 139). Incluso en la primera mitad del siglo XX, las alusiones a las teorías de las emociones en algunos de los textos publicados en revistas, como la Revista de la Universidad de Antioquia o la Revista de Indias, se hacen por intermediación de Bergson — puente, también, entre el pensamiento escolástico y el científico- y no se alude de manera directa a James o a Lange. Igual que sucede con la teoría de la evolución de Darwin, parece haber una censura a la alusión directa a una teoría científica de las emociones ${ }^{7}$.

\section{CONCLUSIONES}

Las palabras tienen sentidos que se complejizan o explican en el transcurso de la historia humana, incluidos aquellos que tienen lugar a causa de los progresos científicos. Un ejemplo claro de ello es el concepto 'tiempo'; su significado sufriría una alteración cuando Einstein publicara los primeros trabajos relativistas. Podría afirmarse, de igual manera, que para conceptos como 'emoción' o 'conciencia' se abrió una perspectiva diferente según las reflexiones científicas. Este tipo de irrupciones lingüísticas dan la posibilidad de mirar al pasado para indagar la forma en que palabras ahora cotidianas y significados ahora obvios serían comprendidos, reinterpretados y expuestos por quienes fueron testigos de su fundación y consolidación. Rastrear la historia de un concepto como 'emoción' demuestra que este ha sido considerado desde diferentes perspectivas. Particularmente su significado sufrió un cambio radical durante el siglo XIX. Para este caso particular, tales transformaciones de registro lingüístico y semántico se fueron

7 Revista Universidad de Antioquía: "Henri Bergson y la filosofía cristiana”, de Félix Henao Botero"; "De la risa”, de Rafael Mesa Duque; "La biogeografía y la evolución”, de Enrique Congote. Revista de Indias: “¿Es la historia un engaño?”, de J. B. S. Haldane. 
depurando a medida que la ciencia llevaba un paso adelante sus indagaciones respecto del ser humano. Para el concepto 'emoción', de hecho, este no puede considerarse un proceso concluido; nuevas ramas de la neurobiología están suscitando modernas variaciones.

Salazar Martínez I

Es clara la participación que tuvieron en el cambio de significado del concepto 'emoción' tanto los filósofos ingleses como los estadounidenses. Su participación activa en el desarrollo científico de campos, como la psicología, la biología y la fisiología, permite entender que haya sido de esa forma. En figuras tan influyentes como Darwin y William James, estuvieron los pilares para la popularización de esa perspectiva. Al entender la profunda importancia que desempeña el cerebro y los sentidos en todo este proceso, se confrontaron perspectivas filosóficas en las que conceptos, como 'emoción', 'conciencia', 'pasiones', 'afecciones' o 'voluntad', eran decisivas para consolidar sus estructuras de pensamiento. Es patente el hecho de que el desarrollo científico fue el motor de la alteración de su condición lingüística al proporcionar una nueva respuesta respecto del cómo, por ejemplo, se produce una emoción. Desde ahí se entiende que no fuera posible para la filosofía y la psicología experimental considerar las emociones en la misma vía en que eran tomadas por la filosofía teológica o moral.

William James no sería el único que plantearía una propuesta respecto de concebir la emoción desde una perspectiva científica, pero sí fue el más influyente. Su esfuerzo, la discusión que abrió con sus teorías al respecto, permitiría que filósofos contrastaran, cuestionaran o intentaran conciliar con ese nuevo ángulo conceptos, hasta ese momento, vitales para entender la mente y el ser humano, como la voluntad, los sentimientos, la conciencia o las pasiones.

No hay registro de la influencia directa de James respecto de la llegada de su pensamiento a Colombia. Es de destacar, sin embargo, el uso que hace Fernando González Ochoa (1930, 1933, 2008) de ciertos criterios propios de la psicofisiología de finales del siglo XIX en sus obras. Sí es posible, al contrario, encontrar de manera directa registro del ingreso del pensamiento de la psicología experimental, con pensadores como Wilhelm Wundt. Y hasta de la filosofía vitalista de Henri Bergson. Cómo cada una de esas corrientes cambió la manera de entender el concepto 'emoción' hace parte de un trabajo futuro.
Es posible pensar la psicología experimental de Wundt como una perspectiva científica aún más concreta de las emociones que la psicofisiología de James, ya que para este último era un desacierto, por ejemplo, considerar la conciencia como un "epifenómeno"; desde este punto de vista la oposición o el impulso que pueda ejercer la conciencia sobre el cuerpo se presenta como impotente ante los acontecimientos. Dar ese paso, para James, significaba pensar el fin de la voluntad.

Considerar el concepto de 'emoción' desde una perspectiva científica implica reconocer que hay ciertos patrones de conducta cuya manifestación está relacionada con la participación dinámica del cuerpo y, con este, el cerebro. En las últimas dos décadas, el avance de la tecnología y las nuevas teorías han permitido poner en primer plano la inquietud por la conceptualización del concepto 'emoción'. Por esto, se reabre una discusión que comenzó con esos primeros esfuerzos de Thomas Brown y la teoría de la expresión de las emociones de Charles Darwin.

\section{REFERENCIAS BIBLIOGRÁFICAS}

1. Bergson, H. (2007). L'essai sur les données immédiates de la conscience. París: Presses Universitaires de France.

2. Brooks, D. (2012). El animal social. Barcelona: Ediciones B.

3. Brown, T. (1820/2010). Thomas Brown: Selected philosophical writings (ed., T. Dixon). Exeter, UK: Imprint Academic.

4. Changeux, J.-P. y Ricoeur, P. (2001). La naturaleza y la norma: lo que nos hace pensar. México: Fondo de Cultura Económica.

5. Congote G., E. (1936). La biogeografía y la evolución. Revista Universidad de Antioquia, 6, 271-274.

6. Costa Carvalho, M. (2012). The bio-philosophical "insufficiency" of darwinism for Henri Bergson's metaphysical evolutionism. Process Studies, 41(1), 133-149.

7. Darwin, C. (1873). The expression of emotions in animals and man. Nueva York: Appleton.

8. Damasio, A. (2010). En busca de Spinoza: neurobiología de las emociones y los sentimientos. Barcelona: Crítica. 
9. Deigh,J. (2014). William James and the rise of the scientific study of emotion. Emotion Review, 6(1), 4-12.

10. Dixon, T. (2012). "Emotion": The history of a keyword in crisis. Emotion Review, 4(4), 338-344.

11. Ekman, P.y Oster, H. (1979). Facial expressions of emotion. Annual Review of Psychology, 30, 527-554.

12. Ekman, P. (1984). Expression and the nature of emotion. En K. R. Scherer y P.Ekman (eds.), Handbook of methods in nonverbal behavior research (pp. 45-135). Nueva York: Cambridge University Press.

13. Ekman, P. (1989). The argument and evidence about universals in facial expressions of emotion. En H. Wagner y A. Manstead (eds.), Handbook of social psuchophysiology (pp. 143-164). Chichester, Inglaterra: Wiley.

14. Ekman, P. (2009). Darwin's contributions to our understanding of emotional expressions. Philosophical Transactions of the Royal Society B: Biological Sciences, 364(1535), 3449-3451.

15. Friedman, B. H. (2010). Feelings and the body: the Jamesian perspective on autonomic specificity of emotion. Biological psychology, 84(3), 383-393.

16. Garrison,J. (2003). Dewey's theory of emotions: The unity of thought and emotion in naturalistic functional "co-ordination" of behavior. Transactions of the Charles S. Peirce Society, 405-443.

17. González, F. (1930). Mi Simón Bolívar (vol. 1). Editorial Cervantes. Recuperado de http:// www.medellindigital.gov.co/Mediateca/repositorio\%20de\%20recursos/Gonz\%C3\%A1lez,\%20 Fernando/Gonzalez\%20Fernando_Mi\%20 Simon\%20Bolivar.pdf

18. González, F. (1995). Los negroides. Medellín: Pontificia Universidad Bolivariana. Recuperado de http://files.jorgegaviria.webnode.es/

19. González, F (2008). Salomé. El remordimiento. Medellín: Eafit.

20. Henao Botero, F. (1935). Henri Bergson y la filosofía cristiana. Revista Universidad de Antioquia, 4, 59-61.

21. James, W. (1884). What is an emotion? Mind, 9, 188-205.
22. James, W. (1890). The principles of psychology. The University of Adelaide Library. Recuperado de https://ebooks.adelaide.edu.au/j/james/william/ principles/complete.html

23. James, W. (1894). The physical basis of emotion. Psychological Review, 1, 516-529.

24. Kahneman, D. (2012). Pensar rápido, pensar despacio. Debate: Barcelona.

25. Kandel, E. (2001). Principios de neurociencia. Madrid: MacGraw-Hill.

26. Koselleck, R. (1993). Futuro pasado: para una semántica de los tiempos históricos. Buenos Aires: Paidós.

27. Koselleck, R. (2010). Historia/historia (trad. e introd. A. Gómez Ramos). Madrid: Trotta.

28. Lange, C. G. (1887). Ueber Gemuthsbewegungen. Eine psycho-physiologische Studie. Leipzig: Theodor Thomas.

29. Lange, C. G. y James, W. (1922). The emotions. Baltimore: Williams \& Wilkins.

30. Matt, S. J. (2011). Current emotion research in history: Or, doing history from the inside out. Emotion Review, 3(1), 117-124.

31. Matt, S. J. y Stearns, P. N. (eds.) (2013). Doing emotions history. University of Illinois Press.

32. Mesa Duque, R. (1936). De la risa. Revista Universidad de Antioquia, 2, 212-215.

33. Montaigne, M. (2003). Ensayos completos / Seguidos de todas sus cartas conocidas hasta el día. Recuperado de http://www.cervantesvirtual. com/obra/ensayos-de-montaigne--0/

34. Paloma, O. y Leonardo, G. (s. f.). El proceso secular de la conciencia psicológica en Colombia (1886-1917) (Tesis de doctorado, Universidad Nacional de Colombia, Colombia).

35. Oviedo, G. L. (2012). Colombian approaches to psychology in the 19th century. History of psychology, 15(4), 291.

36. Palencik, J.T. (2007). William James and the psychology of emotions: from 1884 to the present. Transactions of the Charles $S$. Peirce Society: A Quarterly Journal in American Philosophy, 43(4), 769-786.

I Panorama

I pp. 56-64

I Volumen 9

Número 16 
38. Reddy, W. M. (2001). The navigation of feeling: A framework for the history of emotions. Cambridge University Press.

39. Reisenzein, R. y Stephan, A. (2014). More on James and the physical basis of emotion. Emotion Review, 6(1), 35-46.

Salazar Martínez I

40. Russell, J.A. (1980). A circumplex model of affect. Journal of Personality and Social Psychology, 39(6), 1161.

41. Scherer, K. R. (1981). Speech and emotional states. En J. Darby (ed.), The evaluation of speech in psychiatry. Nueva York: Grune \& Stratton.

42. Scherer, K. R., Banse, R., Wallbott, H. G. y Goldbeck, T. (1991). Vocal cues in emotion encoding and decoding. Motivation and Emotion, 15(2), 123-148.

43. Scherer, K. R. (2000, septiembre). Emotion effects on voice and speech: Paradigms and approaches to evaluation. En Presentation held at ISCA Workshop on Speech and Emotion (vol. 10), Belfast.

44. Scherer, K. R. (2003). Vocal communication of emotion: A review of research paradigms. Speech Communication, 40(1), 227-256.

45. Scherer, K. R. (2005). What are emotions? And how can they be measured? Social Science Information, 44(4), 695-729.

46. Scherer, K. R. (2009). Emotions are emergent processes: they require a dynamic computational architecture. Philosophical Transactions of the Royal Society B: Biological Sciences, 364(1535), 3459-3474.

47. Stearns, P. N. (2014). Obedience and emotion: A challenge in the emotional history of childhood. Journal of Social History, 47(3), 593-611.

48. Taylor, E. (2010). William James and the humanistic implications of the neuroscience revolution: An outrageous hypothesis. Journal of Humanistic Psychology, 50(4) 410-429.

Panorama I

pp. 56-64 I

Volumen 9 |

Número 16 |

Enero-junio I

2015 I

50. Wassmann, C. (2014). Picturesque incisiveness: Explaining the celebrity of James's theory of emotion. Journal of the History of the Behavioral Sciences, 50(2), 166-188.

51. Wierzbicka, A. (2010). The "history of emotions" and the future of emotion research. Emotion Review, 2(3), 269-273.

49. Wassmann, C. (2009). Physiological optics, cognition and emotion: A novel look at the early work of Wilhelm Wundt. Journal of the History of Medicine and Allied Sciences, 64(2), 213-249.
52. Wundt, W.M. (1905). Grundzüge der physiologischen Psychologie. Leipzig: Engelmann. 